\title{
THE DECONFINEMENT TRANSITION FOR QUENCHED SU(2) LATTICE QCD WITH WILSON FERMIONS
}

\author{
J. ENGELS \\ Fakultàt für Physık, Unıversität Bıelefeld, Bıelefeld, Germany \\ and \\ F. KARSCH \\ CERN, Geneva, Switzerland
}

Recelved 13 January 1983

\begin{abstract}
The fermion contribution to the energy density of SU(2) lattice QCD is calculated in the quenched approximation for Wilson fermions as a function of temperature. The technique employed is a high order hopping parameter expansion. We find that the deconfinement temperature is - essentially independent of the quark mass - the same as that earlier determined for the pure gauge field part of SU(2) lattice QCD The critical hopping parameter is estimated from the convergence radius of $\langle\bar{\psi} \psi\rangle$. At least for SU(2), the quantity $\langle\bar{\psi} \psi\rangle$ shows no drastic change in behaviour indicating the exact position of a chiral phase transition.
\end{abstract}

During the last two years MC simulations for pure Yang-Mills systems on the lattice have given strong support to the long standing idea of a phase transition in strongly interacting matter ${ }^{\neq 1}$. It has been shown that these systems undergo a deconfining phase transition [2,3] from a state of confined gluons ("glueball matter") to a state of a gluon plasma with asymptotically free constituents [3]. The introduction of fermions into the theory leads to a corresponding state of free quarks and gluons in the high temperature limit. This was shown to first non-vanishing order in the hopping parameter expansion [4]. The influence of contributions of higher orders in this expansion and the behaviour at lower temperatures, especially in the deconfinement transition region of the gluonic part, are to be investigated in the following. To achieve this we calculate the fermionic part of the energy density $\epsilon^{\mathrm{F}}$ and the quantity $\langle\bar{\psi} \psi\rangle$, which is assumed to be an order parameter for chiral symmetry breaking.

We consider the SU(2) lattice version of QCD with

\#1 For a recent review, see Satz [1].
Wilson fermions. As we shall use the quenched approximation to the theory in the actual Monte Carlo simulations, it is sufficient to take into account only one flavour for the quarks. The euclidean action of the system is given by

$S_{\mathrm{E}}=S^{\mathrm{G}}+S^{\mathrm{F}}$,

with

$$
\begin{aligned}
S^{\mathrm{G}} & =4\left(K_{\beta}^{\mathrm{G}} \sum_{\left\{\mathrm{P}_{\beta}\right\}}\left(1-\frac{1}{2} \operatorname{tr} U_{i j} U_{j k} U_{k l} U_{l l}\right)\right. \\
& \left.+K_{\sigma}^{\mathrm{G}} \sum_{\left\{\mathrm{P}_{\sigma}\right\}}\left(1-\frac{1}{2} \operatorname{tr} U_{i j} U_{j k} U_{k l} U_{l i}\right)\right)
\end{aligned}
$$

for the pure gauge field part of the action and

$$
S^{\mathbf{F}}=\sum_{n, m} \bar{\psi}_{n} Q_{n m} \psi_{m},
$$

where

$$
\begin{aligned}
& Q_{n m}=1-K_{\beta} M_{0, n m}-K_{\sigma} \sum_{\mu=1}^{3} M_{\mu, n m}, \\
& M_{\mu, n m}=\left(1-\gamma_{\mu}\right) U_{n m} \delta_{n, m-\hat{\mu}}+\left(1+\gamma_{\mu}\right) U_{m n}^{\dagger} \delta_{n, m+\hat{\mu}},
\end{aligned}
$$


for the quark-gluon sector. Additional colour and spinor indices have been suppressed in eqs. (4) and (5). The summation over $\mathrm{P}_{\sigma}\left(\mathrm{P}_{\beta}\right)$ in eq. (2) refers to a sum over all plaquettes with links in space-space (space temperature) direction. Notice that the pure gauge field part $S^{\mathrm{G}}$ depends on two couplings $K_{\beta}^{\mathrm{G}}$, $K_{\sigma}^{\mathrm{G}}$ which are functions of the usual coupling constant $g^{2}$ and the parameter $\xi=a_{\sigma} / a_{\beta}[3]$. Here $a_{\sigma}\left(a_{\beta}\right)$ denote the lattice spacings in space (temperature) direction. Similarly the fermionic part, $S^{\mathrm{F}}$, of the action depends on the couplings $K_{\beta}, K_{\sigma}$ which are given by $[4,5]$

$$
\begin{aligned}
& K_{\beta}=\frac{1}{2}[\xi /(3+\xi)] k_{\beta}\left(\xi, g^{2}\right), \\
& K_{\sigma}=\frac{1}{2}[1 /(3+\xi)] k_{\sigma}\left(\xi, g^{2}\right) .
\end{aligned}
$$

In the limit $g^{2} \rightarrow 0$ the functions $k_{\beta(\sigma)}\left(\xi, g^{2}\right)$ approach unity and for $\xi=1$

$K_{\beta}\left(1, g^{2}\right)=K_{\sigma}\left(1, g^{2}\right)=K\left(g^{2}\right)$

is just the usual hopping parameter [6]

The euclidean partition function of the quarkgluon system on a finite $N_{\sigma}^{3} \times N_{\beta}$ lattice is then given by

$Z_{\mathrm{E}}=\int \prod_{\text {links }} \mathrm{d} U \prod_{\text {sites }} \mathrm{d} \bar{\psi} \mathrm{d} \psi \exp \left(-S_{\mathrm{E}}\right)$,

where in thermal direction the bosonic integration variables $U$ obey periodic boundary conditions and the anticommuting spinor fields $\bar{\psi}, \psi$ antiperiodic boundary conditions. The temperature $T=\beta^{-1}$ and volume $V$ of the system are specified by

$\beta=N_{\beta} a_{\beta}, \quad V=\left(N_{\sigma} a_{\sigma}\right)^{3}$.

From eq. (8) one obtains the euclidean energy density $\epsilon_{\mathrm{E}}$

$$
\begin{aligned}
\epsilon_{\mathrm{E}} & =-V^{-1}\left(\partial \ln Z_{\mathrm{E}} / \partial \beta\right)_{V} \\
& =\left(\xi^{2} / N_{\sigma}^{3} N_{\beta} a_{\sigma}^{4}\right)\left(\partial \ln Z_{\mathrm{E}} / \partial \xi\right)_{a_{\sigma}},
\end{aligned}
$$

which after subtraction of the vacuum contribution leads to the physical energy density $\epsilon$. Performing first the derivative in eq. (10) and then integrating over the fermionic degrees of freedom $\bar{\psi}, \psi$ yields the energy density $\epsilon_{\mathrm{E}}$ as a sum of two terms

$\epsilon_{\mathrm{E}}=\epsilon_{\mathrm{E}}^{\mathrm{G}}+\epsilon_{\mathrm{E}}^{\mathrm{F}}$,

with

$$
\begin{aligned}
\epsilon_{\mathrm{E}}^{\mathrm{G}} & =-\left[4 \xi^{2} /\left(N_{\sigma}^{3} N_{\beta} a_{\sigma}^{4}\right)\right] \\
& \times\left[\left(\frac{\partial K_{\beta}^{\mathrm{G}}}{\partial \xi}\right){ }_{a_{\sigma}}\left\langle\operatorname{det} Q \sum_{\left\{\mathrm{P}_{\beta}\right\}}\left(1-\frac{1}{2} \operatorname{tr} U U U U\right)\right\rangle_{U}\right. \\
& \left.+\left(\frac{\partial K_{\sigma}^{\mathrm{G}}}{\partial \xi}\right)_{a_{\sigma}}\left\langle\operatorname{det} Q \sum_{\left\{\mathrm{P}_{\sigma}\right\}}\left(1-\frac{1}{2} \operatorname{tr} U U U U\right)\right\rangle_{U}\right] \\
& \times\left(\langle\operatorname{det} Q\rangle_{U}\right)^{-1}
\end{aligned}
$$

for the gluon part, and

$$
\begin{aligned}
\epsilon_{\mathrm{E}}^{\mathrm{F}} & =-\left[\xi^{2} /\left(N_{\sigma}^{3} N_{\beta} a_{\sigma}^{4}\right)\right] \\
& \times\left[\left(\frac{\partial K_{\beta}}{\partial \xi}\right)_{a_{\sigma}}\left\langle\operatorname{det} Q \operatorname{tr} M_{0} Q^{-1}\right\rangle_{U}\right. \\
& \left.+\left(\frac{\partial K_{\sigma}}{\partial \xi}\right)_{a_{\sigma}}\left\langle\operatorname{det} Q \sum_{\mu=1}^{3} \operatorname{tr} M_{\mu} Q^{-1}\right\rangle_{U}\right]\left(\langle\operatorname{det} Q\rangle_{U}\right)^{-1}
\end{aligned}
$$

for the fermionic part.

In eqs. (12) and (13) $\langle\ldots\rangle_{U}$ denotes expectation values with respect to the gluon field distributions

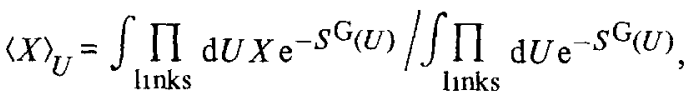

correspondingly the expectation value of $\bar{\psi} \psi$ can be written as

$\langle\bar{\psi} \psi\rangle=\left\langle\operatorname{det} Q \operatorname{tr} Q^{-1}\right\rangle_{U} /\langle\operatorname{det} Q\rangle_{U}$.

The quenched approximation [7] consists now in setting

$\operatorname{det} Q \equiv 1$

in all the formulae. Then as can be seen from eq. (12) the quantity $\epsilon^{\mathrm{G}}$ becomes the energy density of the pure Yang-Mills system, which has been treated in detail elsewhere [3].

To obtain the derivatives $\partial K_{\sigma(\beta)} / \partial \xi$ in $\epsilon^{\mathrm{F}}$ we neglect, as in ref. [4], the $\xi$ dependence of the functions $k_{\sigma(\beta)}\left(\xi, g^{2}\right)$,

$k_{\sigma(\beta)}\left(\xi, g^{2}\right) \simeq k_{\sigma(\beta)}\left(1, g^{2}\right)=8 K\left(g^{2}\right)$.

As a consequence the derivatives become

$\partial K_{\sigma} / \partial \xi \simeq-\left[1 / 2(3+\xi)^{2}\right] k_{\sigma}\left(1, g^{2}\right)$,

$\partial K_{\beta} / \partial \xi \simeq\left[3 / 2(3+\xi)^{2}\right] k_{\beta}\left(1, g^{2}\right)$. 
The actual Monte Carlo calculation was carried out on an isotropic lattice $\left(\xi=1 ; a_{\sigma}=a_{\beta}=a\right)$, so one has only one hopping parameter $K\left(g^{2}\right)$. We thus get from eq. (18)

$\partial K_{\sigma} /\left.\partial \xi\right|_{\xi=1} \simeq-\frac{1}{4} K\left(g^{2}\right)$,

$\partial K_{\beta} /\left.\partial \xi\right|_{\xi=1} \simeq \frac{3}{4} K\left(g^{2}\right)$.

Moreover, in this approximation the vacuum contribution to $\epsilon^{\mathrm{F}}$ becomes zero.

The main problem in the evaluation of both $\epsilon^{\mathrm{F}}$ and $\langle\bar{\psi} \psi\rangle$ is then the inversion of the matrix $Q$. The expectation values $\left\langle\operatorname{tr} M_{\mu} Q^{-1}\right\rangle_{U}$ and $\left\langle\operatorname{tr} Q^{-1}\right\rangle_{U}$ can be computed in an iteration process by making use of the hopping parameter expansion $[8,9]$ of $Q^{-1}$

$Q^{-1}=(1-K M)^{-1}=\sum_{l=0}^{\infty} K^{l} M^{l}$,

where

$M=\sum_{\mu=0}^{3} M_{\mu}$.

The procedure works in principle in the following way: for a given gauge field configuration one starts with the matrix $M_{\mu}$ and successively multiplies with $M$ to obtain $M_{\mu} M^{l}$ after $l$ steps. In each step one takes the trace $\operatorname{tr}\left(M_{\mu} M^{l}\right)$. After reaching the highest order desired, several updates of the gauge fields are made followed by a new determination of the traces, etc. As a result one obtains a hopping parameter expansion for both $\epsilon^{\mathrm{F}}$ and $\langle\bar{\psi} \psi\rangle$. The same method has been used by Hasenfratz and Montvay to get a high order hopping parameter expansion for the hadron spectrum [10].

In the actual Monte Carlo calculations eight rows of the matrices $M_{\mu} M^{l}$ [those belonging to a randomly chosen lattice point, but all colours (2) and spins (4)] were evaluated for $l=1, \ldots, 45$ at a given link configuration. The sum of the diagonal elements of these rows times the number of lattice points was then taken as an estimate for the traces.

Our data were measured on an $8^{3} \times 3$ lattice by averaging over 50 different gauge field configurations at each value of $g^{2}$. Between each estimate of traces 30 new updates of the links were made in order to obtain statistically independent gauge field configu-

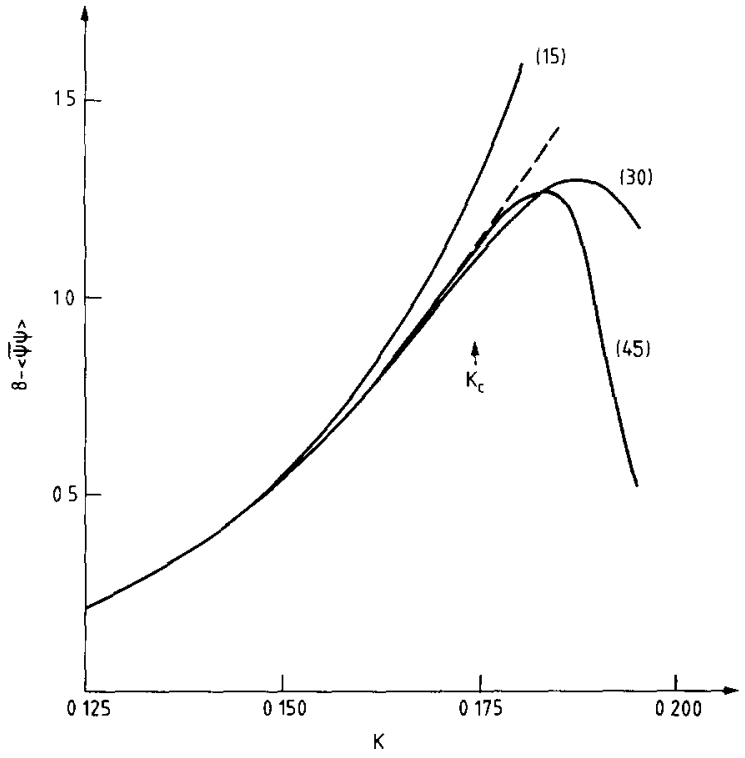

Fig 1 Hopping parameter expansion for the quantity 8 $\langle\bar{\psi} \psi\rangle$ at $4 / g^{2}=219$ truncated after 15,30 and 45 orders versus hopping parameter $K$ Also shown is the [20,20]-Padé approximant to the serres (dashed line) and the location of $K_{\mathrm{c}}$.

rations. Before each trace calculation, we checked that the thermal Wilson loop was positive. If this was not the case, a corresponding transformation of the link matrices was carried out, so that the system was always in the phase, which is connected to the physical continuum limit, where $U \rightarrow 1$ [4] . In fig. 1 we show $\langle\bar{\psi} \psi\rangle$ at $4 / g^{2}=2.19$ as a function of the hopping parameter $K$ for up to 15,30 and 45 orders. Though the coefficients of the $K$ expansion still statistically fluctuate with the number of estimates of the traces, the resulting sum is stable below some critical $K$ value already after 30 trials. Since

$\ln \operatorname{det} Q=-\operatorname{tr} \sum_{l=1}^{\infty} \frac{K^{l}}{l} M^{l}$,

one may determine also the unquenched expectation values with the same method, however many more estimates of $\operatorname{tr} M^{l}$ at each gauge field configuration are then needed because the product of traces $\left(\operatorname{tr} M^{l}\right)$ $\times\left(\operatorname{tr} M^{\prime}\right)$ is required.

The energy density $\epsilon^{\mathrm{F}}$ and $\langle\bar{\psi} \psi\rangle$ still depend on $g^{2}$ 
and $K\left(g^{2}\right)$. As we are working in the quenched approximation $g^{2}$ is related to the lattice spacing $a$ by the pure gauge field renormalization group equation

$a \Lambda_{\mathrm{L}}=\left(11 g^{2} / 24 \pi^{2}\right)^{-51 / 121} \exp \left(-12 \pi^{2} / 11 g^{2}\right)$,

which fixes the temperature $T=\left[N_{\beta} a\left(g^{2}\right)\right]^{-1}$. The hopping parameter $K$ is connected to the quark mass $m_{\mathrm{q}}[9]$ via

$m_{q}=\ln \left[1+\frac{1}{2}\left(1 / K-1 / K_{c}\right)\right]$,

and $K_{\mathrm{c}}=1 / 8$ for a free theory $\left(g^{2}=0\right)$ on an infinite lattice. At finite $g^{2}$, however, both $K$ and $K_{\iota}$ have to be renormalized. For SU(3) $K_{\mathrm{c}}\left(g^{2}\right)$ was determined such that the pion mass vanishes [9]. An alternative approach was suggested by Kawamoto [11], who pointed out that $K_{\mathrm{c}}\left(\mathrm{g}^{2}\right)$ might be the convergence radius for the hopping parameter expansion of $\langle\bar{\psi} \psi\rangle$. Based on weak coupling as well as large $N$ considerations, he conjectures that $\langle\bar{\psi} \psi\rangle$ develops for all finite values of $\mathrm{Ng}^{2}$ a branch cut at that value of $K$ where the pion mass vanishes

$\langle\bar{\psi} \psi\rangle \simeq c_{1}+c_{2}\left(K_{\mathrm{c}}-K\right) \ln \left(K_{\mathrm{c}}-K^{\prime}\right)$.

When this conjecture is true 1 gives an easy way to determine $K_{\mathrm{c}}$. Indeed Padé approxumants to the hopping parameter expansion of $\langle\bar{\psi} \psi\rangle$ show no isolated poles but always pole-zero pairs in the neighbourhood of the real axis, which simulate the cut. As a consequence of eq. (25) we have a pole in the second derivatıve of $\langle\bar{\psi} \psi\rangle$ with respect to $K$. With the help of Padé approximants to the expansion of $\partial^{2}\langle\bar{\psi} \psi\rangle / \partial K^{2}$ we were able to determine the corresponding $K_{\mathrm{c}}\left(g^{2}\right)$. It is shown in fig. 2 . Where they are comparable, these values for $K_{\mathrm{c}}\left(g^{2}\right)$ agree with those determined by Weingarten from the vanushing of the pion mass [12] . In the actual calculation the singularity closest to $K=0$ was not a single pole on the positive real axis, but a pair of complex poles near to the real axis. Since we have a finite lattice in temperature direction, such a behaviour is quite natural. To see this, consider the quark propagator of the free theory. It has a pole for

$\left(1-2 K \sum_{\mu=0}^{3} \cos \left(p_{\mu} a\right)\right)^{2}+4 K^{2} \sum_{\mu=0}^{3} \sin ^{2}\left(p_{\mu} a\right)=0$.

On an infinite lattice eq. (26) leads to a critical value $K_{\mathrm{c}}=1 / 8$ for $p_{\mu}=0$. However, on a lattice which is finite and antiperiodic at the boundaries in the tem-

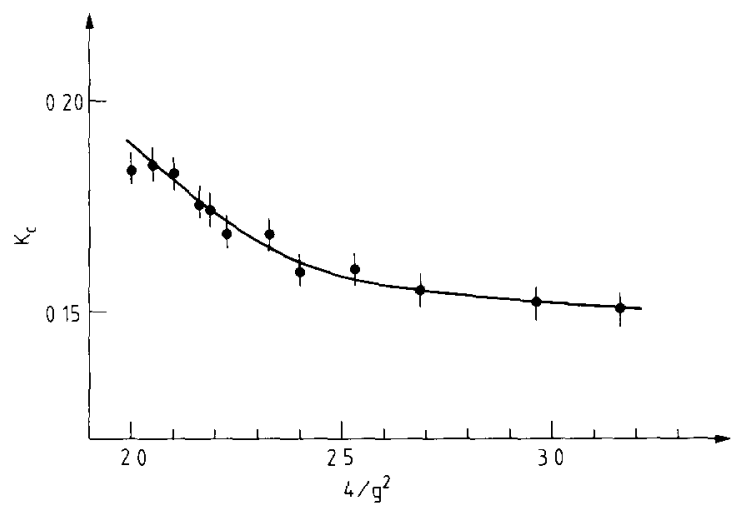

Fig 2. The critical value $K_{\mathrm{c}}$ for the hopping parameter as a function of $4 / g^{2}$ The curve is a fit by eye to the data points.

perature direction the lowest momentum state allowed is given by

$p_{0} a=\pi / N_{\beta}, \quad \boldsymbol{p}=0$.

The propagator has then two complex poles at

$K_{\mathrm{c}}^{N_{\beta}}=\frac{3+\cos \left(\pi / N_{\beta}\right) \pm \mathrm{i} \sin \left(\pi / N_{\beta}\right)}{4\left[5+3 \cos \left(\pi / N_{\beta}\right)\right]}$,

and the convergence radius is, e.g., for $N_{\beta}=3$

$\left|K_{\mathrm{c}}^{N_{\beta}=3}\right|=0.13868$,

which is somewhat larger than the critical value $1 / 8$ for an infinite lattice.

In fig. 3 the fermion energy density $\epsilon^{\mathrm{F}} / T^{4}$ for one quark flavour and massless quarks, i.e., at the critical values $K_{\mathrm{c}}$ (taken from the fit in fig. 2) is shown as a function of the temperature. The straight line in fig. 3 gives the value of $\epsilon^{\mathrm{F}} / T^{4}$ for the free theory at $K_{\mathrm{c}}$ $=K_{\mathrm{c}}^{N_{\beta}}$ on a finite lattice of the same size $\left(8^{3} \times 3\right)$. Notice that due to finite size effects [5] this value is about a factor 4.3 larger than the continuum value for a free gas of massless fermions

$\epsilon_{\mathrm{SB}} / T^{4}=7 \pi^{2} / 30, \quad \mathrm{SU}(2)$.

The energy density $\epsilon^{\mathbf{l}}$ approaches the value of the free theory with increasing temperature. At about $T$ $=100 \Lambda_{\mathrm{L}}$ the limiting curve is already reached after a sudden jump at around $T_{\mathrm{c}} \simeq 40 \Lambda_{\mathrm{L}}$. Thus the fermion energy density shows - in the quenched approxımation - the same behaviour as the energy den- 


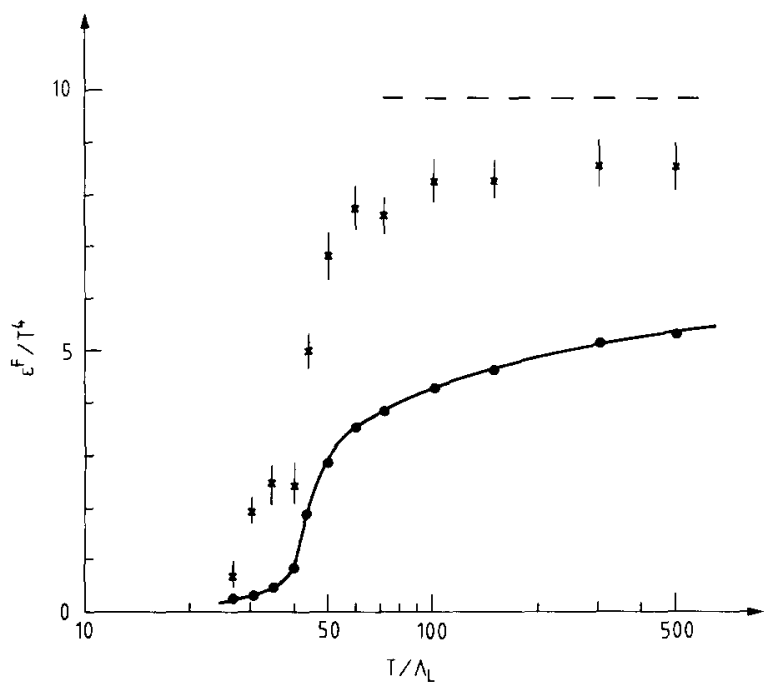

Fig 3. The fermionic contribution $\epsilon^{\mathrm{F}}$ to the energy density of SU(2) lattice QCD divided by the fourth power of the temperature $T$ versus $T / \Lambda_{\mathrm{L}}$ for massless quarks, $1 \mathrm{e}, K=K_{\mathrm{C}}$ (crosses) and massive quarks, 1e, $K=1 / 8$ (full points) Also shown is the high temperature Stefan-Boltzmann limit (dashed line) on a lattice of the same size $\left(8^{3} \times 3\right)$.

sity $\epsilon^{G}$ of the pure Yang-Mills system [3], in particular the deconfinement temperature $T_{\mathrm{c}}$ does not change. The uncertainty in the determination of $K_{\mathrm{c}}$ does not influence the main features of $\epsilon^{\mathrm{F}}(T)$, because a change in $K$ corresponds to a change in the quark mass, which is irrelevant in the high temperature region. Also, the deconfinement temperature does not shift when the quark mass is enhanced. This can be seen from an estimate of the energy density of heavy quarks. From the SU(3) calculations for hadron masses [9] one knows that the hopping parameter for heavy quarks is weakly dependent on $g^{2}$ and that it approaches the value $1 / 8$ from below. Thus, $\epsilon^{\mathrm{F}}$ for $K=1 / 8$ can be considered a reasonable approximation to the energy density of heavy quarks. As can be seen from fig. 3 , there is still a sudden increase at $T_{\mathrm{c}} \simeq 40 \Lambda_{\mathrm{L}}$, but of course the high temperature limit is approached much slower than for massless quarks.

Finally, let us comment on the problem of chiral symmetry restoration. This question is in the case of Wilson fermions particularly complex since chiral symmetry is by construction broken on the lattice. Even a system of non-interacting massless fermions leads to

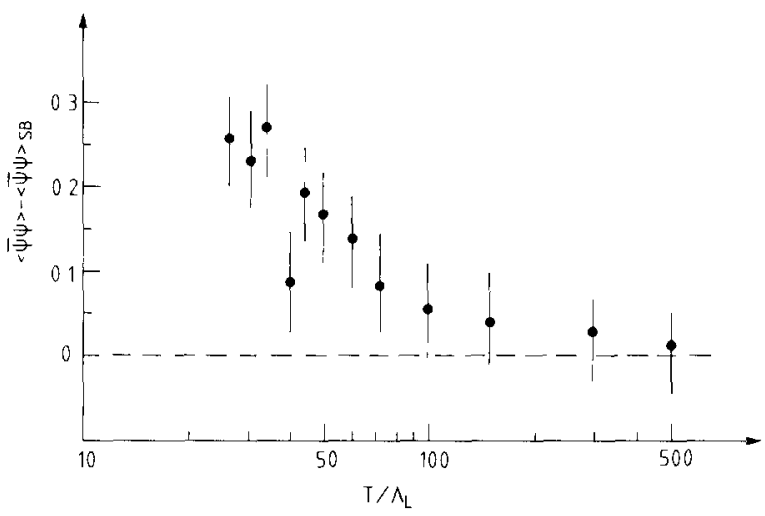

Fig 4 The quantity $\langle\bar{\psi} \psi\rangle-\langle\bar{\psi} \psi\rangle_{S B}$ versus temperature for $K=K_{\mathrm{c}}$ with $K_{\mathrm{c}}$ taken from the fit to the data of fig. 2

a $\langle\bar{\psi} \psi\rangle_{\mathrm{SB}}$ which is not zero at $T=0$. To study chiral symmetry restoration, one would therefore first have to show that $\langle\bar{\psi} \psi\rangle$, after subtraction of the vacuum term, exhibits scaling behaviour and then check at what $T$ it leads to a vanishing expectation value indicating chiral symmetry restoration. This would require a study of $\langle\bar{\psi} \psi\rangle$ on larger lattices with varying extent in temperature direction which will be considered in detail elsewhere. Here we note that if we simply consider

$$
\langle\bar{\psi} \psi\rangle-\langle\bar{\psi} \psi\rangle_{\mathrm{SB}},
$$

as a measure of chiral symmetry for Wilson fermions [4,8], then we see from fig. 4 that for $T \geqslant 100 \Lambda_{L}$, chiral symmetry appears restored. At $T_{\mathrm{c}} \simeq 40 \Lambda_{\mathrm{L}}$, however, expression (31) is still finite, suggesting $T_{\mathrm{c}}$ $<T_{\mathrm{CH}}$. These conclusions agree with the result of Kogut et al. for Susskind fermions [13]. They are also in accord with phenomenological considerations [14], which propose $T_{\mathrm{CH}}$ to be greater than or equal to the deconfinement transition temperature $T_{\mathrm{c}}$. But the introduction of virtual quark loops, i.e., the inclusion of $\operatorname{det} Q$ in the calculation may change the behaviour of $\langle\bar{\psi} \psi\rangle$ considerably.

We thank P. Hasenfratz, I. Montvay and H. Satz for discussions.

Note added When this paper was completed and typed we received a preprint by Kogut et al. [15] , in which they discuss the deconfinement and chiral 
phase transitions for $\mathrm{SU}(2)$ and $\mathrm{SU}(3)$ lattice gauge theories with fermions.

\section{References}

[1] H. Satz, Phys. Rep 88 (1982) 349.

[2] L. McLerran and B. Svetitskı, Phys. Lett. 98B (1981) 195, Phys. Rev D24 (1981) 450; J. Kut1, J Polóny1 and K. Szlachány1, Phys. Lett. 98B (1981) 199

J. Engels, F Karsch, H. Satz and I Montvay, Phys. Lett. $101 \mathrm{~B}$ (1981) 89.

[3] J. Engels, F Karsch, H. Satz and I. Montvay, Nucl. Phys. B205 [FS5] (1982) 545.

[4] J. Engels, F Karsch and H. Satz, Phys. Lett. 113B (1982) 398.

[5] J. Engels, F. Karsch and H. Satz, Nucl. Phys B205 [FS5] (1982) 239

[6] K. Wilson, Phys. Rev D10 (1974) 2445; in New phenomena in subnuclear physics (Erice, 1975), ed A. Z1chichı (Plenum, New York, 1977).
[7] E. Marınari, G. Parıs1 and C. Rebb1, Nucl. Phys. B190 (1981) 734

[8] C B. Lang and H. Nicolai, Nucl. Phys B200 [FS4] (1982) 135

[9] A. Hasenfratz, Z. Kunszt, P. Hasenfratz and C.B Lang, Phys. Lett 110B (1982) 289.

[10] P. Hasenfratz and I. Montvay, Hadron spectrum evidence for size problems on the lattice, Santa Barbara preprint NSF-ITP-82-135 (1982)

[11] N. Kawamoto, Nucl. Phys B190 [FS3] (1981) 617.

[12] D. Weingarten, Phys Lett 109B (1982) 57

[13] J. Kogut, M. Stone, H.W. Wyld, J. Shigemitsu, S.H. Shenker and D.K. Sinclair, Phys. Rev. Lett 48 (1982) 1140.

[14] E.V. Shuryak, Phys. Lett. 107B (1981) 103, Nucl Phys B203 (1982) 140, R.D. P1sarsk1, Phys Lett. 110B (1982) 155.

[15] J. Kogut, M. Stone, H.W. Wyld, W.R. Gibbs, J. Shigemitsu, S.H. Shenker and D.K. Sinclarr, Deconfinement and chiral symmetry restoration at finite temperature in SU(2) and SU(3) gauge theorles, Illınors preprint ILL-(TH)-82-39 (1982). 